Pacific Journal of Mathematics

THE SUM OF TWO INDEPENDENT EXPONENTIAL-TYPE 


\title{
THE SUM OF TWO INDEPENDENT EXPONENTIAL-TYPE RANDOM VARIABLES
}

\author{
E. M. BOLGER
}

Let $X_{1}, X_{2}$ be nondegenerate, independent, exponential-type random variables (r.v.) with probability density functions, (p.d.f.) $f_{1}\left(x_{1} ; \theta\right), f_{2}\left(x_{2} ; \theta\right)$, (not necessarily with respect to the same measure), where $f_{i}\left(x_{i} ; \theta\right)=\exp \left\{x_{i} p_{i}(\theta)+q_{i}(\theta)\right\}$ for $\theta \in(a, b)$ and $p_{i}(\theta)$ is an analytic function of $\theta$ (for $\operatorname{Re} \theta \in(a, b)$ ) with $p_{i}^{\prime}(\theta)$ never equal to zero on $(a, b)$. If $X_{1}, X_{2}$ are neither both normal nor both Poisson type r.v.'s, then $X_{1}+X_{2}$ is an exponential-type r.v. if and only if $p_{1}^{\prime}(\theta)=p_{2}^{\prime}(\theta)$.

2. Lemmas. It follows from Patil's result ([3]) that a r.v. $X$ is of exponential type if and only if the cumulants, $\lambda_{j}(\theta)$, exist and satisfy

$$
\lambda_{j}^{\prime}(\theta)=p^{\prime}(\theta) \lambda_{j+1}(\theta) \quad \text { for } j=1,2,3, \cdots \text {. }
$$

Lehmann ([2], p. 52) has shown that $q(\theta)$ and hence also $\lambda_{j}(\theta)$ are analytic functions of $p(\theta)$. Then $\lambda_{j}(\theta)$ is an analytic function of $\theta$ for $\operatorname{Re} \theta \in(a, b)$.

Let $\lambda_{j, i}(\theta)$ be the $j^{\text {th }}$ cumulant of $X_{i}$ and $\lambda_{j}(\theta)$ the $j^{\text {th }}$ cumulant of $Y$. Then

$$
\begin{aligned}
\lambda_{j}(\theta) & =\lambda_{j, 1}(\theta)+\lambda_{j, 2}(\theta) \\
\lambda_{j, i}^{\prime}(\theta) & =p_{i}^{\prime}(\theta) \lambda_{j+1, i}(\theta)
\end{aligned}
$$$$
\text { for } j=1,2,3, \cdots \text {. }
$$

Let $h_{j}(\theta)=\lambda_{j, 1}(\theta) \lambda_{2,2}(\theta)-\lambda_{j, 2}(\theta) \lambda_{2,1}(\theta)$ and $c(\theta) \equiv \lambda_{2,2}(\theta) / \lambda_{2,1}(\theta)$.

LEMmA 1. If $h_{3}(\theta) \equiv 0$ and if $c^{\prime}(\theta) \equiv 0$, then either $X_{1}$ and $X_{2}$ are both normal or $p_{1}^{\prime}(\theta) \equiv p_{2}^{\prime}(\theta)$.

Proof. Since $h_{3}(\theta) \equiv 0$,

$$
\lambda_{3,2}(\theta)=c(\theta) \lambda_{3,1}(\theta) .
$$

Since $c^{\prime}(\theta) \equiv 0$,

$$
\lambda_{2,2}^{\prime}(\theta)=c(\theta) \lambda_{2,1}^{\prime}(\theta) .
$$

From (3), (4) and (5) it follows that

$$
p_{2}^{\prime}(\theta) \lambda_{3,2}(\theta)=c(\theta) p_{1}^{\prime}(\theta) \lambda_{3,1}(\theta)=p_{1}^{\prime}(\theta) \lambda_{3,2}(\theta) .
$$


If $\lambda_{3,2}(\theta) \equiv 0$, then $\lambda_{3,1}(\theta) \equiv 0$ and $X_{1}, X_{2}$ are both normal. If there is a point $\theta_{0}$ such that $\lambda_{3,2}(\theta) \neq 0$, then there is a neighborhood, $N\left(\theta_{0}\right)$, in which $\lambda_{3,2}(\theta) \neq 0$. For $\theta \in N\left(\theta_{0}\right), p_{1}^{\prime}(\theta)=p_{2}^{\prime}(\theta)$. By analyticity, $p_{1}^{\prime}(\theta)=$ $p_{2}^{\prime}(\theta)$ for $\theta \in(a, b)$.

Lemma 2. If $h_{j}(\theta) \equiv 0$ for $j>2$ and if $c^{\prime}(\theta) \not \equiv 0$, then $X_{1}$ and $X_{2}$ are Poisson type r.v.'s.

Proof. Since $h_{j}(\theta) \equiv 0$,

$$
\lambda_{j, 2}(\theta)=c(\theta) \lambda_{j, 1}(\theta) .
$$

Differentiating (6) and using (3), we get

$$
c(\theta) \lambda_{j, 1}^{\prime}(\theta)+c^{\prime}(\theta) \lambda_{j, 1}(\theta)=p_{2}^{\prime}(\theta) \lambda_{j+1,2}(\theta) .
$$

Then,

$$
c(\theta) p_{1}^{\prime}(\theta) \lambda_{j+1,1}(\theta)+c^{\prime}(\theta) \lambda_{j, 1}(\theta)=p_{2}^{\prime}(\theta) c(\theta) \lambda_{j+1,1}(\theta) .
$$

In particular,

$$
c(\theta) p_{1}^{\prime}(\theta) \lambda_{3,1}(\theta)+c^{\prime}(\theta) \lambda_{2,1}(\theta)=p_{2}^{\prime}(\theta) c(\theta) \lambda_{3,1}(\theta) .
$$

Multiplying (7) by $\lambda_{3,1}(\theta)$ and (8) by $\lambda_{j+1,1}(\theta)$, we find that

$$
c^{\prime}(\theta)\left[\lambda_{2,1}(\theta) \lambda_{j+1,1}(\theta)-\lambda_{3,1}(\theta) \lambda_{j, 1}(\theta)\right]=0
$$

Since $c^{\prime}(\theta) \not \equiv 0$, there is a sub-interval $M$ of $(a, b)$ in which $c^{\prime}(\theta) \neq 0$. For $\theta \in M$,

$$
\lambda_{2,1}(\theta) \lambda_{j+1,1}(\theta)-\lambda_{3,1}(\theta) \lambda_{j, 1}(\theta)=0,
$$

or

$$
\lambda_{j+1,1}(\theta)=\frac{\lambda_{3,1}(\theta)}{\lambda_{2,1}(\theta)} \lambda_{j, 1}(\theta) .
$$

By analyticity, (10) is true for all $\theta \in(a, b)$. Now let $a(\theta)=\lambda_{3,1}(\theta) / \lambda_{2,1}(\theta)$. Then, by (3),

$$
\begin{aligned}
p_{1}^{\prime}(\theta) \lambda_{4,1}(\theta) & =\lambda_{3,1}^{\prime}(\theta)=a^{\prime}(\theta) \lambda_{2,1}(\theta)+a(\theta) \lambda_{2,1}^{\prime}(\theta) \\
& =a^{\prime}(\theta) \lambda_{2,1}(\theta)+a(\theta) p_{1}^{\prime}(\theta) \lambda_{3,1}(\theta) .
\end{aligned}
$$

Since $\lambda_{4,1}(\theta)=a(\theta) \lambda_{3,1}(\theta)$, it follows that

$$
\alpha^{\prime}(\theta) \lambda_{2,1}(\theta)=0 .
$$

So $a^{\prime}(\theta)=0$ and $a(\theta)=d$. Then (10) becomes

$$
\lambda_{j+1,1}(\theta)=d \lambda_{j, 1}(\theta)
$$


This implies

$$
\lambda_{j, 1}(\theta)=d^{j-2} \lambda_{2,1}(\theta)
$$

By (6),

$$
\lambda_{j, 2}(\theta)=d^{j-2} c(\theta) \lambda_{2,1}(\theta)
$$$$
\text { for } j \geqq 2 \text {. }
$$

Now,

$$
\begin{aligned}
& p_{1}^{\prime}(\theta)=\lambda_{1,1}^{\prime}(\theta) / \lambda_{2,1}(\theta), \\
& p_{1}^{\prime}(\theta)=\lambda_{2,1}^{\prime}(\theta) / \lambda_{3,1}(\theta)=\lambda_{2,1}^{\prime}(\theta) / d \lambda_{2,1}(\theta) .
\end{aligned}
$$

So

$$
\lambda_{1,1}(\theta)=d^{-1} \lambda_{2,1}(\theta)+k_{1}
$$

Similarly,

$$
\lambda_{1,2}(\theta)=d^{-1} c(\theta) \lambda_{2,1}(\theta)+k_{2} .
$$

Using (12), (13), (14) and (15), we find that

$$
\begin{aligned}
& \log M_{1}(t ; \theta)=k_{1} t+d^{-2} \lambda_{2,1}(\theta)\left(e^{d t}-1\right) \\
& \log M_{2}(t ; \theta)=k_{2} t+d^{-2} c(\theta) \lambda_{2,1}(\theta)\left(e^{d t}-1\right),
\end{aligned}
$$

where $M_{i}(t ; \theta)$ is the moment generating function corresponding to $f_{i}\left(x_{i} ; \theta\right)$.

This concludes the proof of Lemma 2 .

3. The sum of two independent exponential-type random variables.

THEOREM 1. If $X_{1}, X_{2}$ are neither both normal nor both Poisson type r.v.'s, then $X_{1}+X_{2}$ is an exponential-type r.v. if and only if $p_{1}^{\prime}(\theta)=p_{2}^{\prime}(\theta)$.

Proof. If $p_{1}^{\prime}(\theta)=p_{2}^{\prime}(\theta)$, then if follows from (2) and (3) that

$$
\begin{aligned}
\lambda_{j+1}(\theta) & =\lambda_{j+1,1}(\theta)+\lambda_{j+1,2}(\theta) \\
& =\left[p_{1}^{\prime}(\theta)\right]^{-1} \lambda_{j, 1}^{\prime}(\theta)+\left[p_{1}^{\prime}(\theta)\right]^{-1} \lambda_{j, 2}^{\prime}(\theta) \\
& =\left[p_{1}^{\prime}(\theta)\right]^{-1} \lambda_{j}^{\prime}(\theta) .
\end{aligned}
$$

Conversely, assume $X_{1}+X_{2}$ is an exponential-type r.v.. Then, using $(1),(2)$, and (3), we find that

$$
p^{\prime}(\theta)\left[\lambda_{j, 1}(\theta)+\lambda_{j, 2}(\theta)\right]=p_{1}^{\prime}(\theta) \lambda_{j, 1}(\theta)+p_{2}^{\prime}(\theta) \lambda_{j, 2}(\theta) .
$$

In particular, 


$$
p^{\prime}(\theta)\left[\lambda_{2,1}(\theta)+\lambda_{2,2}(\theta)\right]=p_{1}^{\prime}(\theta) \lambda_{2,1}(\theta)+p_{2}^{\prime}(\theta) \lambda_{2,2}(\theta) .
$$

Multiplying (16) by $\lambda_{2,1}(\theta)$ and (17) by $\lambda_{j, 1}(\theta)$ and then subtracting, we get

$$
\left[p^{\prime}(\theta)-p_{2}^{\prime}(\theta)\right] h_{j}(\theta) \equiv 0 \quad \text { for } j \geqq 2 \text {. }
$$

Now, if for some $j_{0} \geqq 2, h_{j_{0}}(\theta) \not \equiv 0$, then there is a subinterval, $M$, of $(a, b)$ in which $h_{j_{0}}(\theta) \neq 0$. Then, for $\theta \in M, p_{2}^{\prime}(\theta)=p^{\prime}(\theta)$. By analyticity, $p_{2}^{\prime}(\theta)=p^{\prime}(\theta)$ for all $\theta \in(a, b)$. Substitution in (16) yields $p_{1}^{\prime}(\theta)=$ $p^{\prime}(\theta)$ for $\theta \in(a, b)$. If, on the other hand, $h_{j}(\theta) \equiv 0$, for $j \geqq 2$, the result follows from Lemmas 1 and 2 since we assumed that $X_{1}, X_{2}$ are neither both normal nor both Poisson type r.v.'s.

It should be noted that Girshick and Savage [1] proved that if $X_{1}$ and $X_{2}$ are independent identically distributed r.v.'s such that their sum is of exponential-type, then $X_{1}$ and $X_{2}$ are also of exponentialtype.

The following theorem gives necessary and sufficient conditions for the sum of two Poisson-type r.v.'s to be exponential-type.

Theorem 2. If $\log M_{i}(t ; \theta)=C_{i} t+A_{i}(\theta)\left[l^{b_{i} t}-1\right]$, then $X_{1}+X_{2}$ is an exponential-type r.v. if and only if either $b_{1}=b_{2}$ or $p_{1}^{\prime}(\theta)=$ $p_{2}^{\prime}(\theta)$.

Proof. If $X_{1}+X_{2}$ is an exponential-type r.v., then, as in the proof of the preceding theorem,

$$
\left[p^{\prime}(\theta)-p_{2}^{\prime}(\theta)\right] h_{j}(\theta) \equiv 0
$$

for $j \geqq 2$.

Equivalently,

$$
\begin{aligned}
& {\left[\lambda_{j, 1}(\theta) \lambda_{2,2}(\theta)-\lambda_{j, 2}(\theta) \lambda_{2,1}(\theta)\right]} \\
& \quad=p_{2}^{\prime}(\theta)\left[p^{\prime}(\theta)\right]^{-1}\left[\lambda_{j, 1}(\theta) \lambda_{2,2}(\theta)-\lambda_{j, 2}(\theta) \lambda_{2,1}(\theta)\right] \quad \text { for } j \geqq 2 .
\end{aligned}
$$

Since, for $j \geqq 2, \lambda_{j, i}(\theta)=b_{i}^{j} A_{i}(\theta)$, (19) becomes

$$
\left[b_{1}^{j} b_{2}^{2}-b_{2}^{j} b_{1}^{2}\right] A_{1}(\theta) A_{2}(\theta)=p_{2}^{\prime}(\theta)\left[p^{\prime}(\theta)\right]^{-1}\left[b_{1}^{j} b_{2}^{2}-b_{2}^{j} b_{1}^{2}\right] A_{1}(\theta) A_{2}(\theta) .
$$

But $A_{1}(\theta) A_{2}(\theta)>0$, so that

$$
\left[b_{1}^{j} b_{2}^{2}-b_{2}^{j} b_{1}^{2}\right]=p_{2}^{\prime}(\theta)\left[p^{\prime}(\theta)\right]^{-1}\left[b_{1}^{j} b_{2}^{2}-b_{2}^{j} b_{1}^{2}\right] .
$$

Now, if $b_{1}^{j} b_{2}^{2}=b_{2}^{j} b_{1}^{2}$ for all $j \geqq 2$, then $b_{1}^{3} b_{2}^{2}=b_{2}^{3} b_{1}^{2}$, so that $b_{1}=b_{2}$. On the other hand, if, for some $j_{0}, b_{1}^{j_{0}} b_{2}^{2}-b_{2}^{j_{0}} b_{1}^{2} \neq 0$, then $p_{2}^{\prime}(\theta)=p^{\prime}(\theta)$ and it follows that $p_{1}^{\prime}(\theta)=p_{2}^{\prime}(\theta)$.

Conversely, if $p_{1}^{\prime}(\theta)=p_{2}^{\prime}(\theta)$, then $X_{1}+X_{2}$ is an exponential-type r.v. since (1) is satisfied. If $b_{1}=b_{2}$, let 


$$
p^{\prime}(\theta)=\left[A_{1}^{\prime}(\theta)+A_{2}^{\prime}(\theta)\right] / b_{1}\left[A_{1}(\theta)+A_{2}(\theta)\right] .
$$

It is easy to see that (1) is again satisfied.

The author wishes to thank William L. Harkness for his help in the preparation of this paper.

\section{REFERENCES}

1. M. Girshick and L. Savage, Bayes and minimax estimate for quadratic loss functions, Second Berkeley Symposium on Probability and Statistics, University of California Press, Berkeley, 1951, 67-68.

2. E. L. Lehmann, Testing Statistical Hypotheses, John Wiley, New York, 1959.

3. G. P. Patil, A characterization of the exponential-type distribution, Biometrika 50 (1963), 205-207.

Received August 17, 1964, and in revised form February 26, 1965.

BUCKNELL UNIVERSITY 



\section{PACIFIC JOURNAL OF MATHEMATICS}

H. SAMELSON

Stanford University Stanford, California

R. M. BLUMENTHAL

University of Washington

Seattle, Washington 98105

\section{EDITORS}

\author{
*J. DugundJI \\ University of Southern California \\ Los Angeles, California 90007 \\ RICHARD ARENS \\ University of California \\ Los Angeles, California 90024
}

\section{ASSOCIATE EDITORS}

E. F. BeCKENBACH

B. H. NEUMANN

F. WOLF

K. YosidA

\section{SUPPORTING INSTITUTIONS}

UNIVERSITY OF BRITISH COLUMBIA

CALIFORNIA INSTITUTE OF TECHNOLOGY

UNIVERSITY OF CALIFORNIA

MONTANA STATE UNIVERSITY

UNIVERSITY OF NEVADA

NEW MEXICO STATE UNIVERSITY

OREGON STATE UNIVERSITY

UNIVERSITY OF OREGON

OSAKA UNIVERSITY

UNIVERSITY OF SOUTHERN CALIFORNIA
STANFORD UNIVERSITY

UNIVERSITY OF TOKYO

UNIVERSITY OF UTAH

WASHINGTON STATE UNIVERSITY

UNIVERSITY OF WASHINGTON

AMERICAN MATHEMATICAL SOCIETY CHEVRON RESEARCH CORPORATION TRW SYSTEMS

NAVAL ORDNANCE TEST STATION 


\section{Pacific Journal of Mathematics}

Vol. 18, No. 1

March, 1966

Edward Joseph Barbeau, Semi-algebras that are lower semi-lattices ...... 1

Steven Fredrick Bauman, The Klein group as an automorphism group

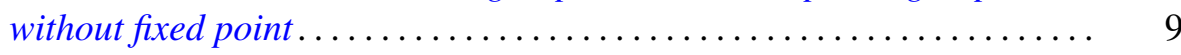

Homer Franklin Bechtell, Jr., Frattini subgroups and $\Phi$-central groups .... 15

Edward Kenneth Blum, A convergent gradient procedure in prehilbert

spaces ............................................

Edward Martin Bolger, The sum of two independent exponential-type random variables ...................................

David Wilson Bressler and A. P. Morse, Images of measurable sets .......

Dennison Robert Brown and J. G. LaTorre, A characterization of uniquely

divisible commutative semigroups........................

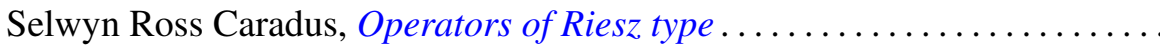

Jeffrey Davis and Isidore Isaac Hirschman, Jr., Toeplitz forms and ultraspherical polynomials ............................

Lorraine L. Foster, On the characteristic roots of the product of certain rational integral matrices of order two ......................

Alfred Gray and S. M. Shah, Asymptotic values of a holomorphic function

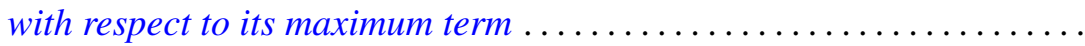

Sidney (Denny) L. Gulick, Commutativity and ideals in the biduals of

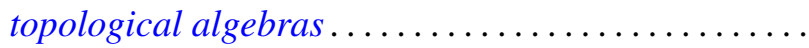

G. J. Kurowski, Further results in the theory of monodiffric functions

Lawrence S. Levy, Commutative rings whose homomorphic images are self-injective .

Calvin T. Long, On real numbers having normality of order $k$....

Bertram Mond, An inequality for operators in a Hilbert space. ...

John William Neuberger, The lack of self-adjointness in three-point boundary value problems ........................

C. A. Persinger, Subsets of $n$-books in $E^{3}$

Oscar S. Rothaus and John Griggs Thompson, A combinatorial problem in the symmetric group ............................... 175

Rodolfo DeSapio, Unknotting spheres via Smale .................. 179

James E. Shockley, On the functional equation

$$
F(m n) F((m, n))=F(m) F(n) f((m, n)) \ldots \ldots \ldots
$$

Kenneth Edward Whipple, Cauchy sequences in Moore spaces ... 